\title{
THE INTERRUPTION USED BY THE HOST AND GUESTS IN SARAH SECHAN TALK SHOW ON NET TV
}

\author{
ANNISA ANGGIA AYU MAGHFIRO ${ }^{1}$, MEISURI ${ }^{2}$, SITI AISAH GINTING ${ }^{3}$ \\ ${ }^{123}$ UNIVERSITAS NEGERI MEDAN
}

\begin{abstract}
This research aims to identify the types and reasons of interruption uttered by host and the guests in Sarah Sechan talk show. To answer the objectives of the research, the researcher used conversation analysis approach. The data were the utterances that contain the interruption from the conversation in Sarah Secham Talk Show Net TV. The findings showed that there were four types of interruption employed by the host and guests, i.e. simple interruption, overlap interruption, silent interruption and butting-in interruption. From 56 data found in the talk show, simple interruption had the biggest number of occurrence with $37.50 \%$. Simple interruption was the most appearing type in this talk show because each guest in every episode mainly gives his/her floor to the interrupter, which is the host, Sarah sechan even though his/her utterance was disrupted. The smallest number of interruption's type was butting-in interruption with only 5.35\%. The host or the guests seldom do butting-in interruption because they give a chance to the interrupter to deliver his/her message. To decide the reason of interruption, the writer looked at the context of the discussion between the host and the guests. The common reason was Completing with $35.71 \%$ and the other reason was showing agreement, seeking clarification, correcting, breaking up, and reject some point. From all of this reason the writer conclude that the interruptions in this talk-show were not violation.
\end{abstract}

Keywords: conversation analysis; interruptionn; Sarah Sechan Talk

\section{INTRODUCTION}

As social creature, people need to communicate in order to maintain their relationship and to give or gain information from others. In these interactions, conversations occur between two or more people in which one person in a particular time plays as an informant or speaker, and the other plays as a listener. As a mode of communication, conversation is therefore deemed more effective than any other communicative devices since it can transfer messages clearly via verbal and non-verbal ways at once. For that reason, conversation becomes the most common form of communication that makes up the majority of human communication.

Due to the importance of conversation in human social interaction, a great deal of attention has been devoted by a number of researchers to the analysis of conversation. In the beginning of 1970s, a sociologist Harvey Sacks and his two colleagues, Emmanuel A. Schegloff 
and Gail Jefferson, began to introduce a new approach to the study of conversation which they called Conversation Analysis (CA) (Liddicoat, 2007: 4).

As a matter of fact, the starting point for CA research is in the analysis of turn-taking mechanism. Turn-taking is a system that regulates the alternation of speakers during conversation (Yule, 1996: 72). In any conversation, turn-taking is inevitable and evidently noticeable as, at any time in conversation, interlocutors switch roles as speakers with each other in order to exchange information. In accordance with this, turn-taking is considered to be the most fundamental feature of talk-in-interaction, and therefore discovering how turn-taking operates in conversation is a principal requirement for Conversation Analysis research. The investigation into the organization of turn-taking allows the researchers to recognize what interlocutors undertake when conversing with one another, including how they distribute turns in a systematic order and how they exploit the turns to accomplish particular conversational goals.

On any occasion, the course of conversation will run smoothly and effectively if the communicative participants implement the rules of turn-taking. The basic rule of turn-taking is that interlocutors speak one at a time, that is to say, no more than one person can hold the floor in a certain period of time (Sacks et al. 1974: 700). In practices, nevertheless, interlocutors may depart from the usual turn-taking norm. A next speaker can possibly begin his/her speech at a point when the transfer of speakership is inappropriate, namely that s/he initiates speaking when the current speaker is still in the progress of producing his/her utterance. As a consequence, the current speaker's turn might be disrupted as the other speaker intrudes on his/her unfinished turn. In this case, a violation of the turn-taking norm takes place, and a common term to refer to that violation is interruption.

Zimmerman and West (in Tannen, 1994: 57) explain that the violation occurs when a new speaker attempts to grab the floor despite the fact that the ongoing speaker does not plan to give up his/her turn yet. For this reason, interruption is regularly considered rude and offensive since the interrupters, i.e. the persons who interrupt, seem to be domineering and show disregard for the others' right to talk. As the result, interruption is particularly thought of as always being negative and unfavorable. In certain circumstances, nonetheless, interruption can be positive and advantageous. The interrupters might intend to display cooperation with the ongoing speakers via their interruptions. In reference to this understanding, the researcher was motivated to conduct research on interruption to reveal how it usually occurs and what interactive effects it has especially in Talk Show.

The researcher was interested in analyzing this case because many unique opinions in the society have been existed about the interruption is being called violence in conversation. 
Larasati (2014) states that interruptions often occur in an informal setting between people who have a close relationship. They also can without doubt interrupt another person, especially to people who are younger than them. Anindya (2014) also found that in an interview there was a big possibility of doing interruption. It was because there are some question that the interviewer prepares to be asked in a limited time. Meanwhile, Hartono (2013) concluded that when people often called interruption as violation in conversation because it causes the conversation not to run smoothly, is not completely true. She says that we should know the social distance or relationship that is used by the participants to know whether interruption is violation or not.

Based on previous description, some scholars have conducted research relate to interruption in many different setting. Those are movie conversation, talk show, interview program and in ordinary conversation. Therefore, this study was decided to investigate Sarah Sechan Talk Show in relation to the phenomenon of interruption. Sarah Sechan, as the host might be share feelings of closeness and familiarity to her guest, also tend to freely alternate in speaking and overlook the ways they take turns. So interruption in here could never be avoided. Regardless of the kinship concern, additionally, other matters such as age and social status possibly affect the production of interruption by the host and guests. Indeed, this study provided some insight and understanding not only about the interruption itself, but also take a deep about the types and reasons of interruption that is used by the host and guests in Sarah Sechan Talk Show. Conversation is one of ways used by society to interact each other's. Conversation does not only use the linguistic code, but also other tools, such as intonation, body language, silence, and others. In addition, conversation as a system used to connect between person and other person. However, conversation is more than preserving relationship, but also shows the way speakers to cooperate with other speaker.

In a conversation, the speaker and the listener always change position where the speaker is the listener and the listener is the speaker. It is called turn taking. Turn taking is a fundamental study in a conversation. Normally, in a conversation, a person talks and another person listens. The system happens vice versa for both parties, so each person has right to talk and to listen. The transition between the parties usually occurs without any gap or overlap because it happens naturally; therefore, in natural and spontaneous conversation, turn taking may not always succeed because there's always the situation when the disturbance occurred. Turn taking irregularities can happen because they cut the conversation without giving chance to the speaker to finish his or her words. Zimmerman \& West (1975:114) divide the types of turn-taking irregularities in a conversation into "interruption and overlap". To define interruption is rather complicated because it depends on complex factors, such as the status of speaker and the culture of the place. Understanding interruption is not limited to culture and 
dominance, but it is beyond that. Interruption can also be seen from various points of view, including the time when the interruption occurs and the point where the interruption appears.

Tannen (1990) gives a rather different reason because she also consider other variables such as situation, topic, and speaker's right. She states that "Interruption is not only a matter of violation in conversation but also the individual's rights". For example when the second speaker asks for a glass of water because he or she feels thirsty and interrupts the first speaker, it is not kind of interruption because the speaker cuts the conversation is not interrupt. Tannen also states that interruption is not a violation as long as the topic does not change, for example assent terms such as 'Yes', 'Okay' do not mean to interrupt the conversation, on the other hand, those term are used to make clear to the speaker that the listener has taken in and understand the previous message. They also serve to establish the listener's ongoing availability, and they commit him or her to attend to the speaker's next utterance.

\section{METHODOLOGY}

This research employed a descriptive qualitative method since the object of this research was a social phenomenon such as everyday conversation. Qualitative method does not prove something right or wrong, but explains the reason behind the problem. Moreover, giving point of view from experiences and knowledge obtained was what the researcher tried to do in making the thesis. Therefore, the result might be different from one person to other because of external factors, such as culture, religion, and childhood experience influencing the research process. However, quantitative method is also used as an additional method to present the data in the form of numbers and percentage to create a clearer explanation about the relation between the types and the reasons of interruption in the talk show. To analyze the data, the researcher used textual analysis to interpret the data which were in the form of verbal signs.

After collected and trascribed the data into a writting form, the next step was analyzed the data which following into steps as follows :

1. Organizing and identifying the data from the video

2. Re-watching the video and re-reading the transcript to gain more information about the data.

3. Categorizing the data by classifying them into their respective types and reasons based on the following table : 
Table 1. Types of Interruption

\begin{tabular}{cc}
\hline No & Types of Interruption \\
\hline 1 & Simple Interruption \\
2 & Overlap Interruption \\
3 & Butting- In Interruption \\
4 & Silent Interruption \\
\hline
\end{tabular}

Table 2. Reasons of Interruption

\begin{tabular}{cc}
\hline No & Reasons of Interruption \\
\hline 1 & Showing Agreement \\
2 & Correcting \\
3 & Seeking Clarification \\
4 & Rejecting \\
5 & Completing \\
6 & Topic Change \\
\hline
\end{tabular}

4. Tabulating the frequency of each types and reasons of Interruption

5. Converting the frequency of each types and reasons of Interruption into percentages by formula

6. Interpreting the result by giving an explanation in the form of narrative description based on the theoretical framework

7. Reporting the findings and the discussion and adding points of conclusion.

\section{FINDINGS and DISCUSSIONS}

The data were clasified according to the types and reasons of interruption in Sarah Sechan Talk Show. The total data collected in this research were 56 data. From 4 types and 6 reasons of interruption, they're all existed in this talkshow. The following table showed more information about the frequency of the occurrence related to interruptions.

\begin{tabular}{cccc}
\multicolumn{4}{c}{ Table 3. The Frequency Types of Interruption } \\
\hline No & Types of Interruption & Frequency & Percentage \\
\hline 1 & Simple Interruption & 21 & 37.50 \\
2 & Overlap Interruption & 17 & 30.35 \\
3 & Butting-In Interruption & 3 & 5.35 \\
4 & Silent interruption & 15 & 26.78 \\
& TOTAL & $\mathbf{5 6}$ & \\
\hline
\end{tabular}

The table above showed that there were four type of interruptions; simple interruption, overlaps interruption, butting-in interruption, and silent interruption. The most highest frequency was overlap interruption. Between the host and guest used simple interruption which 
appeared 21 times in two episodes. Then, overlap interruption was the second highest frequency which occured 17 times that produced by the the host and guest of Sarah Sechan Talk Show in two episodes. The next type was silent interruption occurs 15 times. The lowest frequency was butting-in interruption produce by the participants which appeared only 3 times in two episodes.

Table 4. The Frequency Reasons of Interruption

\begin{tabular}{cccc}
\hline No & Reasons of Interruption & Frequency & Percentage \\
\hline $\mathbf{1}$ & Showing Agreement & 14 & 25 \\
$\mathbf{2}$ & Correcting & 5 & 8.92 \\
$\mathbf{3}$ & Seeking Clarification & 12 & 21.42 \\
$\mathbf{4}$ & Reject Some Point & 2 & 3.57 \\
$\mathbf{5}$ & Completing & 20 & 35.71 \\
$\mathbf{6}$ & Topic Change & 3 & 5.35 \\
\hline \multicolumn{5}{c}{56} \\
\hline
\end{tabular}

Table above showed the classification the reasons of interruptions. There are seven reasons based on Wardhaugh's theory ; Showing agreement, Asking for help, seeking clarification, reject some point, completing and topic change/ breaking up. The highest frequency was a completing. It appeared 20 times in two episodes of Sarah Sechan Talk Show in NET TV. The second position was showing aggreement which appeared 14 times in two episodes. The third position was seeking clarification with 12 occurrences. The fourth and fifth position was correcting which occured 5 times and topic change which occurred 3 times. Then, the lowest frequency was rejecting occurred 2 times in two episodes of Sarah Sechan Talk Show.

\section{CONCLUSIONS AND SUGGESTIONS}

After analyzing all the data, it could be concluded that:

1. All the four types of interruptions that consist of simple, overlap, butting-in, and silent interruption appear in the talk show. The types of interruption found are simple interruption (21 times or $37,50 \%$ ), overlaps interruption (17 times or $30,35 \%$ ), silent interruption (15 times or 26,78\%), and butting-in interruption (3 times or 5,35\%).

2. There were six reasons that appeared in this talk show: completing, showing agreement, seeking clarification, correcting, topic change and rejecting. Completing got the biggest percentage with $35.71 \%$. The last reason was rejecting with only 2 occurrence (3.57\%). Completing was underlie the most choice of the reason of interruption because both of the host and guests seems to be have close relationship or at the very least, they know what their counterparts have in their minds. Even if their assumptions regarding their counterparts' judgment is wrong, they are confident enough that they will not be accused or judged wrongly because they share equal power and are in the situation where it is acceptable to interrupt each other. 
This study expected the reader to understand the types of interruption and the reasons why it was occurred. By increasing knowledge of interruption, the reader were expected to be able to distinguish different kinds of interruptions that usually appear in their daily conversations. As the results of this study that interruption does not necessarily serve negative and intrusive functions but also fulfills functions which are cooperative and even neutral. It was suggested to the future researchers and the students of Applied Linguistics who interested to carry out this similar study are expected to be able to explore other aspects related to the phenomenon of interruption, for instance the relationship between interruptions and certain social variables such as status, age, gender, etc., the influence of cultures or personalities on the occurrence of interruption and the characteristics of interruption on the basis of prosodic features. In addition, the researcher suggests the common readers are better to avoid doing interruptions during conversation, especially those which are competitive and unhelpful, in order to achieve smooth interactional exchanges.

\section{REFERENCES}

Beattie, G. W. (1982). Turn-taking and Interruption in Political Interviews: Margaret Thatcher and Jim Callaghan Compared and Contrasted". Semiotica, 39-1/2, pp. 93-114.

Liddicoat, J Anthony. (2007). Introduction to Conversation Analysis. London:Continuum.

Sacks, Schegloff, \& Jefferson. (1978). A simplest systematics for the Organization of Turn-taking for Conversation. In Language Vol. 50 no 4.

Tannen, D. (1990). You just don't understand. New York: William Morrow and Company.

Wardhaugh, R. (1985). How conversation works. Oxford: Blackwell.

Wardhaugh, R. (2006). An introduction to sociolinguistics. Oxford: Blackwell.

Yule, G. (1996). Pragmatics. Oxford: Oxford University press. 1996.

Zimmerman, D. \& West, C. (1975). Sex Roles, Interruptions and Silences in Conversation. In B. Thorne, \& N. Henley (Eds.), Language and Sex: Difference and Dominance (pp. 105-29). Massachusetts: Newbury. 